\title{
LEHRWERKBEGLEITENDE ONLINE - ÜBUNGEN FÜR DEUTSCHLERNEN - DIDAKTISCHER MEHRWERT ODER ERFOLGREICHE KOMMUNIKATION MARKETINGSTRATEGIE?
}

\section{ONLINE VJEŽBE NJEMAČKOG JEZIKA - DODANA DIDAKTIČKA VRIJEDNOST ILI USPJEŠNA KOMUNIKACIJSKA MARKETINŠKA STRATEGIJA?}

\author{
Andreja Retelj \\ Philosophische Fakultät, Universität Ljubljana, Ljubljana, Slowenien \\ Filozofski fakultet, Sveučilište u Ljubljani, Ljubljana, Slovenija
}

Abstract

Der Beitrag setzt sich zum Ziel lehrwerkbegleitende Online-Übungen unter vier Aspekten zu analysieren: Quantitativer Aspekt, Übungsformate, Förderung

der Sprachfertigkeiten und Taxonomie der Lernziele. Das Korpus besteht aus 820 Online-Übungen, die als Zusatzmaterial zu sieben unterschiedlichen Lehrwerkpaketen auf den Stufen A1, A2, B1, insgesamt zu 21 Lehrwerken dienen. Die Analyse der Online-Übungen deutet darauf hin, dass trotz der großen Anzahl der Online-Übungen überwiegend grammatische Strukturen und Wortschatz gefördert werden, was Hand in Hand mit den niedrigsten Taxonomiestufen geht, die mit Online-Übungen erreicht werden. Lehrwerkbegleitende Online-Übungen ermöglichen bisher noch keine vielfältigen Lernwege, denn es wurden nur vier unterschiedliche Übungsformate identifiziert.

\section{Einführung}

Dass die heutige Welt fast komplett digital verläuft, ist eine Tatsache. Jede Information, die man braucht, ist nur einen Klick entfernt und kann ortsunabhängig und in sehr kurzer Zeit im Internet abgerufen werden. Für Wissbegierige ist die Möglichkeit etwas Neues ISSN 1330-0067
Sažetak

Cilj znanstvenog istraživanja prikazanog u ovome radu bio je analizirati popratne online vježbe u 4 aspekta: kvantitativni aspekt, oblici vježbi, poticanje jezičnih vještina i taksonomija ciljeva učenja. Korpus se sastoji od 820 online vježbi, koje služe kao dodatni materijali za sedam različitih paketa udžbenika na razinama $\mathrm{A} 1, \mathrm{~A} 2$, B1, ukupno za 21 udžbenik. Analiza online vježbi pokazuje da se usprkos velikome broju online vježbi pretežno potiču gramatičke strukture i rječnik, što ide ruku pod ruku s najnižim razinama taksonomije, koje se ostvaruju pomoću online vježbi. Popratne online vježbe zasad još ne omogućuju raznolike načine učenja jer su ustanovljena samo četiri različita oblika vježbi. zu lernen wahrscheinlich noch nie in der Geschichte so einfach und nah gewesen wie in der heutigen digitalen Medienwelt /1/. Was bedeutet aber dieses digitale Medienangebot für den schulischen Fremdsprachenunterricht? Noch immer ist nicht ganz klar, inwieweit der heutige Fremdsprachenunterricht digital 
verläuft, wie die Potenziale des digitalen Medienangebots ausgenutzt werden, was für einen didaktischen Mehrwert die digitalen Lernmaterialien besitzen und nicht zuletzt, welchen Einfluss die Digitalisierung auf den Unterricht in der Zukunft haben wird.

Einerseits stellt das Internet Lernenden und Lehrenden eine unendliche Palette an authentischen Materialien zu jedem denkbaren Thema zur Verfügung; es werden stets neue Webseiten, Lernprogramme und Apps entwickelt, die Lernenden ermöglichen, ihr Wissen online oder offline $\mathrm{zu}$ erweitern, $\mathrm{zu}$ vertiefen und an ihre Bedürfnisse anzupassen /2/. Andererseits zeigt aber die Schulpraxis, dass Lehrwerke für den Fremdsprachenunterricht noch nicht komplett an Bedeutung verlieren und noch immer ein fester Bestandteil des Unterrichts sind. Jedoch arbeiten Schulbuchverlage intensiv an ihrem Angebot und bereichern es durch neue digitale Lernmaterialien.

Bestand ein modernes Lehrwerkkomplett noch vor wenigen Jahren aus einem Lehrwerk, einem Arbeitsbuch, einem Lehrerhandbuch und Kassetten oder CDs mit Tonaufnahmen, so werden heute immer häufiger auch eine elektronische Version des Lehrwerks oder eine E-Version mit zusätzlichen interaktiven Komponenten, zweisprachige Glossare, DVDs mit Filmsequenzen, vorbereitete Materialien für Whiteboards, Apps für Vokabeltraining, zusätzliche Arbeitsblätter zum Downloaden und/oder Ausdrucken und zahlreiche lehrwerkabhängige Online-Übungen, vorbereitete Moodle-Kursräume, Quiz in Kahoo und Softwareprogramme zur Unterrichtsplanung für Lehrende angeboten.

Die meisten großen Schulbuchverlage investieren viel Geld und Engagement, um Schritt mit der globalen Digitalisierung zu halten und versuchen Lehrwerke moderner, attraktiver und zeitgemäß herzustellen. Das Angebot wird ständig um zusätzliche digitale Materialien bereichert. In den letzten Jahren ist es fast $\mathrm{zu}$ einem Muss geworden, dass Lehrwerke auch Online-Übungen anbieten, die den Lernenden ermöglichen, mit verschiedenen
Endgeräten, sei es Computer, Handy oder Tablet, selbstständig oder im Unterricht zu lernen. Da diese Lernmaterialien meistens als zusätzliche Lehrwerkkomponente angeboten werden, wird ihnen in der Lehrwerkforschung nur wenig Aufmerksamkeit geschenkt.

Angefangen mit einer Übersicht über den Stand der heutigen digitalen Lernmaterialien sollen in diesem Beitrag lehrwerkbegleitende OnlineÜbungen, die auf den offiziellen Webseiten der Schulbuchverlage zugänglich sind, unter den vier Aspekten Quantität, Förderung der Sprachfertigkeiten, Übungsformate und Taxonomiestufen unter die Lupe genommen werden.

\section{DIGITALE LERNMATERIALIEN - EINE KURZE ÜBERSICHT}

Seit der Verbreitung des Computer-Assisted Language Learning (kurz CALL) sind wir Zeugen einer enormen Entwicklung auf diesem Gebiet /3/. Folglich unterliegen auch digitale Lernmaterialien zum Fremdsprachenlernen einer rasanten Entwicklung. Einen Überblick findet man z.B. bei Mitschian /4/ und Szerszeń /5/. Eine sehr detaillierte und aufschlussreiche Übersicht über Aufgabenorientierung im Bereich E-Learning bieten Biebighäuser, Zibelius und Schmitt an /6/. Schon mit dem Web 1.0 standen vor allem den Lehrenden immer mehr digitale Materialien zur Verfügung, mittels derer sie den Fremdsprachenunterricht bereichern konnten. So entstanden die ersten Softwareprogramme, meistens auf verschiedenen Datenträgern, und später mit der Verbreitung des Internets Webseiten oder Lernpakete online.

Die ersten lehrwerkbegleitenden oder lehrwerkunabhängigen Webseiten zum Fremdsprachenlernen waren vor allem statisch und eindimensional, das heißt, sie waren readonly Materialien. Anbieter mussten die htmlSprache beherrschen, um Webseiten $\mathrm{zu}$ erstellen und über technische Dispositionen wie z.B. einen Server verfügen. Nutzer konnten mit diesen Inhalten nicht interagieren, das heißt, sie konnten die Inhalte nicht verändern. Zwar entstand dadurch eine enorme Quelle von 
Materialien, die von den Lehrkräften im Unterricht eingesetzt wurden und für Abwechslung im Unterricht sorgen konnten, aber aus fremdsprachendidaktischer Sicht waren sie noch immer nicht ganz zufriedenstellend, da sich in diesem Angebot meist geschlossene Übungsformate befanden, die ein vorprogrammiertes Feedback ermöglichten. Solche Übungen wurden vorwiegend mit Autorenprogrammen wie z.B. Hotpotatoes erstellt. Das Hauptziel solcher Übungen war das Einüben von grammatischen Strukturen und Vokabeln.

Mit dem Web 2.0 begann eine ganz neue Ära der Kommunikation und damit auch neuere Möglichkeiten für das Fremdsprachenlernen. Mittlerweile kann jeder digitale Materialien auf sehr einfache Weise selbst erstellen, existierende verändern, adaptieren und eigenen Bedürfnissen anpassen. Man kann mit anderen Lernenden kommunizieren, Informationen austauschen, kollaborieren, reflektieren und mit verschiedenen Web 2.0 Tools eigene vielfältige Inhalte produzieren. Diese Möglichkeiten werden nicht mehr nur von Lehrenden benutzt, was ganz wichtig ist, auch Lernende sind auf diese Weise Hersteller eigener „Produkte“ geworden. Sie können ihre eigenen Bilder, Texte, Video-, Audioaufnahmen mit wenig technischem Aufwand erstellen und veröffentlichen. So entstehen dynamische Materialien wie z.B. Wikis, Pod- und Vodcasts, Blogs und ein fast unzählbares Angebot an diversen Apps zum Fremdsprachenlernen, die vor allem von enthusiastischen Lehrkräften im Unterricht eingebunden werden. Der wachsende Einsatz von Computern und anderen Endgeräten wie Handys und Tablets im Unterricht fordert auch Schulbuchverlage dazu auf, ihr Angebot an digitalen Lernmaterialien ständig zu erweitern.

Das Web 3.0, das als intelligentes Web bezeichnet wird, entwickelt sich in die Richtung der künstlichen Intelligenz, des maschinenunterstützten Lernens, wobei Informationen in semantische Beziehungen zusammengeführt werden, die von Computern verstanden, interpretiert und sortiert werden. Während das Web 2.0 syntaktisch orientiert ist, ISSN 1330-0067 liegt der Fokus beim Web 3.0 auf der Semantik. Welche Neuerungen das Web 4.0 bringen wird, ist noch unklar. Szerszeń /7/ stellt fest, dass die aktuellen Materialien für das E-Learning hauptsächlich Lehrerhandlungen unterstützen. Das stellt zwar zweifellos eine Bereicherung für den DaF-Unterricht dar, jedoch bleibt $\mathrm{zu}$ wünschen, dass die E-Lernmaterialien auch einen positiven Einfluss auf das Lernen und die Lernenden hätten.

\section{ANALYSE DER ONLINE-ÜBUNGEN}

\section{Forschungsfragen}

Das Ziel der Analyse der Online-Übungen war es Daten zu erheben, die eine empirisch fundierte Antwort auf folgende Fragen ermöglichen:

- Was für einen quantitativen Beitrag leistet das kostenlose digitale Zusatzmaterial?

- Um welche Übungsformate handelt es sich bei den Online-Übungen?

- Welche Sprachfertigkeiten werden mit Online-Übungen gefördert?

- Welcher Taxonomiestufe nach Bloom können Online-Übungen zugeordnet werden?

\section{Forschungsmethodologie}

Zur Durchführung der Analyse wurde eine deskriptive und kausal-nichtexperimentelle Methode ausgewählt. Zuerst wurden die Lehrwerke mit Online-Übungen ausgesucht, danach wurden die Online-Übungen hinsichtlich der Kriterien Interaktivität, Sprachfertigkeit, Taxonomiestufen in vier Phasen analysiert. Die Analyse der OnlineÜbungen verlief in vier Phasen: In der ersten Phase der Analyse wurden die Anzahl der Übungen, die Zugänglichkeit und die visuelle Struktur der Online-Übungen analysiert. In der zweiten Phase wurden die Übungen unter dem Aspekt der Übungsformate unter die Lupe genommen. Alle Übungen wurden nach der Lernertätigkeit kategorisiert, und zwar wurde der Frage nachgegangen, was die Lernenden tun müssen, um eine bestimmte Übung lösen

Coden: IORME7 
zu können. Die dritte Phase der Analyse umfasste die Aufteilung der Online- Übungen hinsichtlich der Förderung der Sprachfertigkeiten und die vierte Phase stellt die Kategorisierung der Online- Übungen nach Stufen der Bloom'schen Taxonomie dar.

\section{Korpus und Zugänglichkeit der lehrwerkbegleitenden Online-Übungen}

Das Korpus unserer Untersuchung bilden Online-Übungen zu den Lehrwerken Ideen 1, Ideen 2, Ideen 3 (Hueber Verlag), Themen aktuell 1, Themen aktuell 2, Themen aktuell 3 (Hueber Verlag), Alles stimmt 1, Alles stimmt 2, Alles stimmt 3 (Rokus Klett Verlag), Studio express A1, Studio express A2, Studio express $B 1$ (Cornelsen Verlag), Optimal A1, Optimal A2, Optimal B1 (Klett-Langenscheidt), Klasse! A1, Klasse! A2 (Klett Verlag) und Panorama A1, Panorama A2, Panorama B1 (Cornelsen). Zu den Lehrwerken Alles stimmt 4 und Ideen 3 wurden zur Zeit der Analyse auf den Web-Seiten von den Verlagshäusern keine Online-Übungen angeboten; das Lehrwerk Klasse B1 ist noch nicht erschienen. In die Analyse wurden Online-Übungen von Lehrwerken einbezogen, die in slowenischen Mittelschulen für 15- bis 19jährige Lernende verwendet werden $/ 8 /$.

Beim Eintrag des jeweiligen Lehrwerknamens in eine beliebige Suchmaschine erscheint zumeist die offizielle Webseite des eingegebenen Lehrwerks als erster Treffer. Eine leicht zu merkende Adresse der Webseite mit Online-Übungen gibt es allerdings nicht, da diese nur ein Teil des gesamten Lehrwerkangebots darstellen.

Bei dem Lehrwerk Optimal wird der Bereich der Online-Übungen als Online-Material bezeichnet und fällt durch die Schriftgröße und optische Hervorhebung auf der Webseite sofort ins Auge. Dieser Bereich besteht aus zwei Teilen, und zwar aus Online-Übungen und Arbeitsblättern bzw. Kopiervorlagen, die als PDF-Format heruntergeladen werden können. Mit einem Klick auf die Online-Übungen öffnet sich eine neue Unterseite und die Lernenden können zwischen den Niveaus A1, A2 und B1 wählen, die gleichzeitig auch den Lehrwerken im Papierformat entsprechen. Die OnlineÜbungen sind innerhalb der im Lehrwerk vorhandenen Lektionen konzipiert. Mit einem Klick auf eine bestimmte Lektion öffnet sich eine neue Unterseite mit Übungen. Die Lernenden bekommen Informationen darüber, dass die Übungen als Online-Projekte konzipiert sind, die innerhalb von 30 Minuten gelöst werden sollen, zuhause oder in der Schule bearbeitet werden können und dass die Links regelmäßig überprüft werden.

Online-Übungen, die auf der Webseite gelöst werden können, sind nur für die Niveaus A1 und B1 erstellt, für das Niveau A2 sind jedoch nur Übungen im PDF-Format vorhanden. Bei der Analyse der Aufgaben und des Feedbacks hat sich herausgestellt, dass das Auswertungssystem nicht funktioniert, darum konnte die Analyse nicht weitergeführt werden. Auch werden die Online-Übungen dieses Lehrwerks nicht mehr aktualisiert oder modernisiert.

Ursprünglich wurde das Lehrwerk Studio d in die Analyse einbezogen, das in Slowenien von vielen Schulen im DaF-Unterricht benutzt wird. Noch während der Untersuchung wurde die Seite mit den Online-Übungen nicht mehr aktualisiert und letztendlich gesperrt. Da die in der Untersuchung analysierten OnlineÜbungen nicht mehr zugänglich sind, wurde der Entschluss gefasst, dieses Lehrwerk aus der Analyse auszuschließen. Als Ersatz diente uns dann Studio express. Auf der Webseite des Lehrwerks Studio express sind Online-Übungen zu finden, die interaktive Übungen genannt werden. Sie sind kostenlos erhältlich und die Nutzer müssen nur den Namen oder die ISBN des Lehrwerks oder einen Webcode angeben. Sie sind in aus dem Lehrwerk bereits bekannte Lektionen unterteilt, jedoch eher zum Testen als zum Üben gedacht. Die Lernenden bekommen auch Informationen darüber, welche Fertigkeit bzw. Sprachhandlung mit der Übung gefestigt wird. Es werden Online-Übungen $\mathrm{zu}$ den Lehrwerken auf den Niveaus A1, A2 und B1 angeboten.

Das Lehrwerk Ideen hat ein ähnliches Konzept wie bereits dargestellt. Auch hier stehen den 
Lernenden Online-Übungen zur Verfügung, und zwar für die Lehrwerke Ideen 1 und Ideen 2, was den Niveaus A1 und A2 entspricht. Für das Niveau B1 bzw. für das Lehrwerk Ideen 3 bietet der Verlag keine Online-Übungen an. Die vorhandenen Online-Übungen sind in die aus dem Lehrwerk bekannten Lektionen unterteilt. Die Lernenden finden auf der Webseite auch Informationen über die Anzahl der Übungen in jeder Lektion.

Auch das Lehrwerk Themen aktuell bietet im Bereich Lernen Online-Übungen an. Es öffnet sich eine neue Unterseite, auf der zu jeder Lektion aus Band 1 (Niveau A1) und Band 2 (Niveau A2) Online-Übungen verfügbar sind. $\mathrm{Zu}$ jeder Lektion wird auch die Anzahl der Übungen angegeben. Zu Band 3 (Niveau B1) gibt es auch hier keine Online-Übungen. Die Autoren bieten Aufgaben als Webrecherche an, die als PDF-Format zur Verfügung stehen und didaktisierte Links $\mathrm{zu}$ externen Seiten enthalten.

$\mathrm{Zu}$ den Online-Übungen des für den slowenischen Kontext entwickelten Lehrwerks Alles stimmt gelangt man über die für Lehrende und Lernende konzipierte Webseite des Verlags. Ohne Registrierung sind nur OnlineÜbungen für Band 1 (Niveau A1) zugänglich. Benötigt wird allerdings ein Login mit EmailAdresse und Benutzernamen. Die OnlineÜbungen für Band 2 und 3 sind zwar auch kostenlos, jedoch nur für registrierte Benutzer zugänglich. Die erstellten Online-Übungen sind eher als Test am Ende jeder Lektion denn als begleitende Übungen zum Lehrwerk zu verstehen, da die Konzipierung jede Antwort mit einem Punkt bewertet und keine genaue Übersicht zu den einzelnen Übungen darbietet. Die Ergebnisse des Tests können die Lernenden auch per Email erhalten. Beim Zählen der Übungen wurden einzelne Aufgabentypen in Betracht genommen und nicht die einzelnen Items, wie sie dargeboten werden.

Im aktuellsten Lehrwerk Klasse! ist der Bereich mit Online-Übungen ganz einfach zu finden, da er optisch mit großer Schrift hervorgehoben ist. Neben den Online-Übungen gibt es für jede Lektion auch ein Quiz mit Kahoot und eine App
- phase6 - zum Vokabeltraining. Zur Zeit der Analyse standen nur Band 1 und 2 zur Verfügung.

Die lehrwerkbegleitenden Online-Übungen zum Lehrwerk Panorama befinden sich auf der offiziellen Webseite des Lehrwerks unter den Zusatzmaterialien, ähnlich wie beim Lehrwerk Studio express vom selben Verlag. Die Aufteilung nach Einheiten wie im Lehrwerk ist benutzerfreundlich und übersichtlich. Die Übungen können online bearbeitet oder als Test in einem Gesamtpaket auf die Festplatte gespeichert werden.

Zusammenfassend kann festgestellt werden, dass lehrwerkbegleitende Online-Übungen ausgewählter Lehrwerke auf den offiziellen Webseiten der Verlage zugänglich und meistens benutzerfreundlich durch eine visuelle Hervorhebung leicht zu erkennen sind. Alle Online-Übungen folgen demselben Konzept wie die Übungen im Lehrwerk selbst. Sie sind in Lektionen unterteilt und durch sofortiges Feedback wird den Lernenden ein selbstständiges Üben ermöglicht.

\section{Resultate der Analyse von Online-Übungen und Diskussion}

\section{Aspekt Quantität der Online-Übungen}

Der erste Schritt der Analyse der OnlineÜbungen ergibt einen Überblick über die Zahl der Online-Übungen für jedes Lehrwerk. Aus Tabelle 1 ist ersichtlich, dass alle Lehrwerke, die in der Analyse berücksichtigt wurden, zahlreiche Online-Übungen kostenlos zur Verfügung stellen. Für das Sprachniveau A1 bieten alle Verlage Online-Übungen an. Für das Niveau A2 wurden in allen ausgewählten Lehrwerken Online-Übungen erstellt, außer in Optimal A2. Zwar gibt es in diesem Lehrwerk auch Übungen, die eine Online-Arbeit verlangen, aber da sie nur im PDF-Format vorliegen, ermöglichen sie kein Feedback. Aus diesem Grund konnten sie nicht in die Analyse miteinbezogen werden. Für das Niveau B1 werden nur von drei Anbietern OnlineÜbungen zur Verfügung gestellt. In Tabelle 1 wird die Zahl der Online-Übungen zu bestimmten Lehrwerken je nach Sprachniveau 
nach dem Gemeinsamen Europäischen Referenzrahmen (GER) dargestellt. Es ist evident, dass alle Lehrwerke über zahlreiche
Übungen verfügen, mit fast viermal mehr Online-Übungen für jedes Niveau überragt Studio express jedoch alle anderen.

\begin{tabular}{|c|c|c|c|}
\hline $\begin{array}{c}\text { Online-Übungen zu den } \\
\text { Lehrwerken }\end{array}$ & Niveau A1 & Niveau A2 & Niveau B1 \\
\hline Studio express & 356 & 309 & 337 \\
\hline Optimal & 71 & Keine Online-Übungen & 52 \\
\hline Ideen & 72 & 72 & Keine Online-Übungen \\
\hline Themen aktuell & 61 & 59 & Keine Online-Übungen \\
\hline Alles stimmt & 68 & 57 & 109 \\
\hline Klasse! & 60 & 60 & Keine-Online Übungen \\
\hline Panorama & 128 & 89 & 82 \\
\hline
\end{tabular}

Tabelle 1: Anzahl der Online-Übungen auf den Niveaus A1, A2 und B1

Wie aus Tabelle 1 ersichtlich ist, ist das digitale Angebot zu jedem Lehrwerk wenigstens für zwei Stufen nach GER vorhanden und zwar stehen den Lernenden zahlreiche Übungen zur Verfügung. Im DaF-Unterricht in der Schule können sie in diesem Maße aufgrund der begrenzten Stundenzahl gewiss nicht eingesetzt werden; lehrwerkbegleitende Online-Übungen können von den Lernenden selbstständig sowie zeit- und ortsunabhängig bearbeitet werden, was gewiss von großem Vorteil ist.

\section{Aspekt Übungsformate}

Im zweiten Schritt wurde jede Online-Übung anhand der von den Lernenden verlangten Tätigkeit eingeordnet. Es wurde der Frage nachgegangen, was Lernende tun müssen, um die Aufgabe zu lösen. Zusätzlich interessierte uns noch, ob es sich um Übungen oder um Aufgaben handelt. Die Analyse des OnlineMaterials ergab nur vier verschiedene Übungsformate, die in Tabelle 3 dargestellt werden.

\begin{tabular}{|l|l|}
\hline Aufgabentyp & Tätigkeit der Lernenden \\
\hline Auswählen & $\begin{array}{l}\text { Lernende wählen eine Antwort im Drop-Down-Menü. } \\
\text { Lernende wählen eine Antwort aus dem Angebot aus. (Multiple Choice-Aufgabe). }\end{array}$ \\
\hline Verbinden & $\begin{array}{l}\text { Lernende verbinden zwei zusammenpassende Elemente, indem sie ein Element zu dem } \\
\text { anderen mit der Maus nach links, nach rechts, nach oben, nach unten ziehen. } \\
\text { Lernende verbinden zwei zusammenpassende Elemente, indem sie auf ein Element und } \\
\text { dann auf das dazu passende Element klicken. }\end{array}$ \\
\hline Einordnen & $\begin{array}{l}\text { Lernende bilden mit Wort- oder Satzteilen die richtige Reihenfolge, indem sie einzelne } \\
\text { Elemente nach links, nach rechts, nach oben oder nach unten ziehen. }\end{array}$ \\
\hline Ergänzen & $\begin{array}{l}\text { Lernende füllen die vorbereiteten Lücken oder Kreuzworträtsel aus, indem sie einzelne } \\
\text { Buchstaben, Wörter, Sätze oder einen längeren Text mit der Tastatur eingeben. }\end{array}$ \\
\hline
\end{tabular}

Tabelle 2: Aspekt Übungsformate

Obwohl Online-Übungen optisch schön und modern wirken, ist die Variation der Übungen nicht vielfältig. Stets werden vier Übungsformate eingesetzt, die sich zwar visuell unterscheiden, jedoch sehr ähnliche Lernwege haben. Es kann festgestellt werden, dass Online-Übungen im Vergleich zu den Übungen und Aufgaben im Lehrwerk selbst sogar nur für wenig Übungsvielfalt sorgen. 
Abbildung 1: Beispiel für das Übungsformat Auswählen:

Lies die Fragen und wähle die richtige Antwort aus.
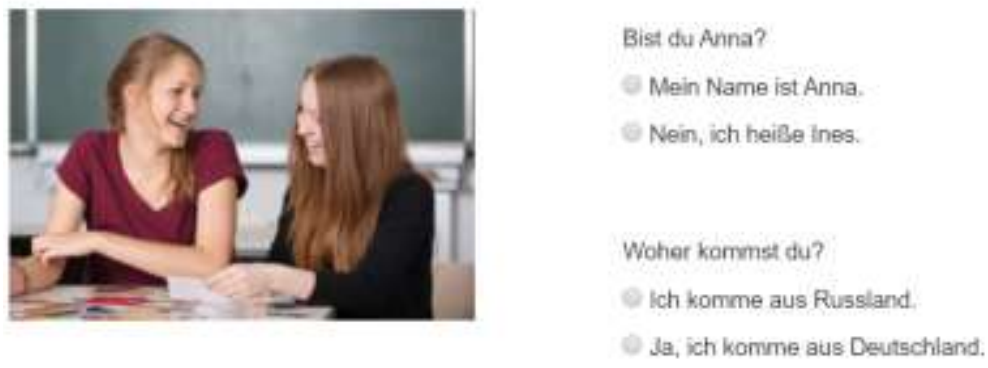

Wer ist das?

Das ist Marta.

2. Ja, ich bin Tim.

Fotografierst du gern?

Ich spiele gem Computer.

O Ja, ich fotografiere sehr gern!

Quelle: Klasse A1

Abbildung 2: Beispiel für das Übungsformat Verbinden

$$
\begin{gathered}
\text { Es liegt auf dem Boden. die Spüle } \\
\text { Hier kann man sich selbst ansehen. } \quad \text { das Sofa } \\
\text { Man muss es gießen. das Bild }
\end{gathered}
$$

Für viele Personen ist das der Platz für Bücher. _ _ die Pflanze

Hier liegt oder hängt oft die Kleidung. _ das Regal Es hängt oft an der Wand. $\quad$ der Teppich Hier können zwei oder drei Personen sitzen. _ der Spiegel Hier wäscht man nach dem Kochen und Essen.

Quelle: Panorama A2 


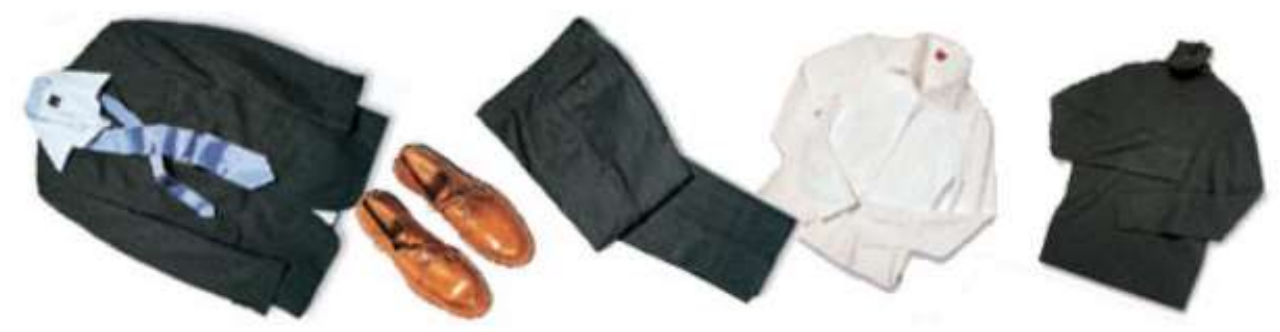

\begin{tabular}{lll}
\hline \multicolumn{2}{c}{ Ordnen Sie die Wörter. } \\
\hline \hline Fertig! & Neustart & Tipp \\
\hline
\end{tabular}

Männer nie tragen $\square$ oder Kleider Röcke

Quelle: Themen aktuell, Band 2, Lektion 1

Abbildung 4: Beispiel für das Übungsformat Ergänzen

Relativpronomen im Genitly. Verbinden Sie die Satze wie im Beispiel.

1. Meine Nachbarin kommt aus Polen. Lch passe manchmal auf ihre Kinder aut.

Meine Nachbarin, auf deren Kinder ich manchmal aufpasse, kommt aus Polen.

2. Herr Özdemir geht im Jult in Rente. Ich kaufe oft in seinem Lebensmittelgeschäft ein

3. Heute steht ein Artikel uber Frau Tran in der Zeitung, In ihrem Frisorsalon sind noch zwei Ausbildungsplátze frei Ober

4. Mein Freund lvan fuhlt sich hier in Bremen zu Hause. Seine Eltern kommen aus der Ukraine

5. Die Meiers reisen zum ersten Mal nach Indien. Thr Sohn atbeitet in Neu Delhi.

6. Mein neuer Chet kommt aus Korea Seine Kinder besuchen hier die internationale Schule 
Quelle: Studio express B1

Eine solche Kategorisierung ergibt einen Überblick über die Vorgehensweise aus technischer Sicht bzw. weist darauf hin, um was für eine Interaktion es sich handelt. Da nur vier verschiedene Kategorien der Lernertätigkeit identifiziert wurden, könnte diese Klassifizierung den Lehrenden und vor allem den Programmierern Informationen dazu liefern, dass identifizierte Tätigkeiten der Lernenden nicht wirklich vielfältig und für Lernende anspruchsvoll sind. Eigentlich kann von neuen Möglichkeiten und einer Entwicklung kaum die Rede sein, da sich technisch gesehen die Art und Weise, wie diese Übungen bearbeitet werden und was Lernende dabei leisten müssen, nicht wesentlich von den Übungen unterscheidet, die schon vor 15 Jahren mit dem Autorenprogramm Hotpotatoes erstellt werden konnten. Obwohl allgemein gerne von einer drastischen Entwicklung des digitalen Lernangebots gesprochen wird, kann diese für den Bereich der lehrwerkbegleitenden OnlineÜbungen nicht bestätigt werden. Die OnlineÜbungen, die in die Analyse miteinbezogen wurden, weisen die meisten Merkmale des Web 1.0 auf. Eine Adoption der Materialien für eigene Bedürfnisse, Kommunikation, kollaboratives Lernen usw. lässt sich bei diesen Übungen noch nicht erkennen. eine Abwechslung im Fremdsprachenunterricht sorgen, doch von einer langfristigen Motivation aufgrund der Aufgabenformate oder einer Veränderung der Lernwege kann nicht wirklich die Rede sein. Der Vorteil von solchen Online-Übungen besteht vor allem im automatischen Feedback, das bei den Drill-Übungen von Nutzen sein kann. Ein sinnvoller Einsatz von OnlineÜbungen im DaF-Unterricht ist gerade in den Übungsphasen $\mathrm{zu}$ sehen, die auf die Automatisierung einer grammatischen Struktur oder das Vokabellernen zielen.

\section{Aspekt Förderung der Sprachfertigkeiten}

Im dritten Schritt wurde jede Online-Übung aus der Sicht der Entwicklung der Sprachfertigkeiten untersucht. Dabei wurden 820 Online-Übungen je nach Lernziel in die Kategorien Sprechen, Schreiben, Lesen, Hören und Wortschatz mit Grammatik eingeordnet. Es wurde die Entscheidung getroffen die Teilfertigkeiten Wortschatz und Grammatik als eine Kategorie zu behandeln, da eine Trennung zwischen den beiden nicht immer eindeutig bzw. in vielen Fällen nicht sinnvoll war. Die Anzahl der Online-Übungen unter dem Aspekt Förderung der Sprachfertigkeiten ist aus Tabelle 3 ersichtlich.

Derartige Übungen wirken auf die Lernenden vielleicht kurzfristig motivierend, weil sie für

\begin{tabular}{|c|c|c|}
\hline Sprachfertigkeit & Zahl der Übungen & Prozentanteil \\
\hline Sprechen & 11 & $1,4 \%$ \\
\hline Schreiben & 15 & $1,8 \%$ \\
\hline Hören & 47 & $5,7 \%$ \\
\hline Lesen & 56 & $6,8 \%$ \\
\hline Wortschatz und Grammatik & 691 & $84,3 \%$ \\
\hline Gesamt & $\mathbf{8 2 0}$ & $\mathbf{1 0 0} \%$ \\
\hline
\end{tabular}

Tabelle 3: Aspekt Förderung der Sprachfertigkeiten

Wie erwartet dient die große Mehrheit der Online-Übungen $(84,3 \%)$ zum Üben von Wortschatz und Grammatik. Hierbei handelt es sich ausschließlich um geschlossene Übungsformate mit einer vorprogrammierten ISSN 1330-0067
Lösung. Die Lernenden werden gleich nach dem Lösen der Aufgaben über ihren Wissensstand informiert. Beide Teilfertigkeiten werden sowohl isoliert als auch im Kontext, angebunden an die Lektionsthematik, geübt.

Coden: IORME7 
Alle vier Übungsformate, die identifiziert wurden (Auswählen, Ergänzen, Verbinden und Einordnen), sind vertreten.

Übungen, die gezielt das Leseverstehen fördern, stehen mit einem wesentlich kleineren Prozentanteil $(6,8 \%)$ an zweiter Stelle. Es handelt sich meistens um kürzere Texte und geschlossene Übungen, bei denen die Lernenden die gesuchten Antworten meistens durch zwei Tätigkeiten eingeben und zwar durch Auswählen und Ergänzen. Das OnlineMaterial verfügt zwar über viel mehr Übungen, bei denen kürzere Texte gelesen werden sollten, jedoch unterliegen diese Übungen meistens dem Ziel Wortschatz oder Grammatik zu festigen. Im Bereich Leseverstehen ist die kleinere Anzahl der Übungen ein wenig überraschend, denn geschlossene Übungsformate sind zum Üben des Leseverstehens ziemlich gut geeignet. Ein Grund dafür könnte darin liegen, dass Lernende auf unterschiedlichen Bildschirmen lesen können, was eine weitere technische Optimierung verlangt. Ein weiterer Grund könnte aber auch sein, dass gute Übungen zum Leseverstehen nicht sehr einfach $\mathrm{zu}$ konzipieren sind.

Eine ähnliche Tendenz wie beim Leseverstehen lässt sich auch beim Hörverstehen beobachten. Nur 5,7 \% der Übungen fördern das Hörverstehen. Etliche Online-Übungen beinhalten zwar auch Audio-Aufnahmen, jedoch sind die Übungen zumeist zur Unterstützung des Vokabellernens oder Aussprachetrainings konzipiert.

Nur ein sehr kleiner Anteil an Online-Übungen fördert beide produktiven Fertigkeiten, Sprechen $(1,4 \%)$ und Schreiben $(1,8 \%)$. Im Bereich Sprechen sind das Übungen, bei denen Lernende ihre Antworten mit verschiedenen Funktionen aufnehmen und sie danach anhören können. Für diese Übungen gibt es auch kein automatisches Feedback. Dasselbe gilt für Schreibaufgaben, die meistens auf einzelne Sätze beschränkt sind, wobei die Lernenden ein Feedback für die Rechtschreibung bekommen können. Ein so kleiner Anteil an Übungen, die produktive
Fertigkeiten fördern, war nach der Feststellung der Übungsformate, die bei Online-Übungen vorkommen, schon zu erwarten. Da sich Online-Übungen fast ausschließlich der geschlossenen Übungsformate bedienen, wo ein sofortiges computerunterstütztes Feedback möglich ist, sind Übungen zur Förderung des Sprechens und Schreibens eher eine Seltenheit.

Zusammengefasst kann festgestellt werden, dass Online-Übungen größtenteils ein Ziel verfolgen und das ist die Förderung des Fremdsprachenlernens im Bereich Vokabelund Grammatiklernen. Alle anderen Fertigkeiten werden wesentlich weniger berücksichtigt oder sogar fast ausgeschlossen. Die Analyse der Online-Übungen unter dem Aspekt Förderung der Sprachfertigkeiten weist darauf hin, dass der Einsatz von OnlineÜbungen größtenteils zum Vokabel- und Grammatiklernen sinnvoll ist. Alle anderen Sprachfertigkeiten finden viel $\mathrm{zu}$ wenig Beachtung und werden in den untersuchten Lehrwerken anhand von Online-Übungen nicht ausreichend gefördert.

\section{Aspekt Taxonomie der Lernziele}

Der letzte in unsere Untersuchung eingeschlossene Aspekt ist die Taxonomie der Lernziele. Dabei bedienten wir uns der Taxonomiestufen nach Bloom /9/ und ordneten diesen alle 820 Online-Übungen hinsichtlich der Lernziele zu. Die Tabelle 4 zeigt die Aufteilung der Online-Übungen hinsichtlich der Lernziele. Der größte Teil der OnlineÜbungen (72,8\%) deckt die niedrigste Stufe ab, das ist das Wissen. Von den Lernenden wird überwiegend erwartet, ihr Wissen wiederzugeben bzw. $\mathrm{zu}$ reproduzieren. Vorausgesetzt wird, dass sie vor allem Vokabeln oder grammatische Phänomene auswendig gelernt haben und dieses erworbene Wissen in einer Online-Übung aufzeigen können. Das ist keineswegs überraschend, denn die meisten Online-Übungen verlangen von den Lernenden das Reproduzieren des Wortschatzes bzw. einer grammatischen Struktur. Mit 17,6 \% aller Online-Übungen belegt die Stufe Verständnis den zweiten Platz. Dazu wurden alle Online-Übungen gezählt, bei 
denen vom Lernenden erwartet wird, etwas zu erklären, zu deuten, einzuordnen oder $\mathrm{zu}$ übersetzen. Der Taxonomiestufe Anwendung wurden 6,5\% der Aufgaben zugeordnet, welche auf der Voraussetzung basieren, dass die Lernenden ihre Kenntnisse anwenden, begründen und interpretieren. In die drei höheren Taxonomiestufen, auf denen von den Lernenden komplexes Wissen und

\begin{tabular}{|c|c|c|}
\hline Taxonomiestufen nach Bloom & Zahl der Übungen & Prozentanteil \\
\hline Wissen & 596 & $72,8 \%$ \\
\hline Verständnis & 145 & $6,5 \%$ \\
\hline Anwendung & 53 & $1,9 \%$ \\
\hline Analyse & 15 & $1,0 \%$ \\
\hline Synthese & 9 & $0,2 \%$ \\
\hline Beurteilung & 2 & $\mathbf{1 0 0} \%$ \\
\hline
\end{tabular}

Tabelle 4: Aspekt Aufteilung der Online-Übungen in die Taxonomiestufen nach Bloom

\section{Fazit und Ausblick}

Das Ziel des Beitrags war lehrwerkbegleitende Online-Übungen nach vier Aspekten zu analysieren und zwar: Quantität der OnlineÜbungen, Übungsformate, Förderung der Sprachfertigkeiten und Taxonomie der Lernziele, wobei auf die Taxonomiestufen nach Bloom zurückgegriffen wurde.

Zusammenfassend kann zum Aspekt Quantität festgestellt werden, dass Online-Übungen zu einem festen Bestandteil des Gesamtangebots eines Lehrwerks geworden sind und aus quantitativer Sicht einen umfangreichen Teil des gesamten Lehrwerksangebots einnehmen. $\mathrm{Zu}$ jedem Lehrwerk wird eine gesonderte Webseite erstellt, die den Lernenden zahlreiche Online-Übungen für selbstständiges Lernen zur Verfügung stellt. Die Anzahl der OnlineÜbungen ist von Lehrwerk zu Lehrwerk unterschiedlich. Die größte Anzahl an OnlineÜbungen ist bei nahezu allen Lehrwerken für die Stufe A1 konzipiert, dann zeigt sich eine leicht sinkende Tendenz bis hin zu sogar fehlenden Online-Übungen auf dem Niveau B1.

Um einen klaren Überblick über das Angebot der Online-Übungen zu ermöglichen,
Kompetenzen erwartet werden, wie z. B. analysieren, gliedern, gegenüberstellen, konstruieren, optimieren, beurteilen, vergleichen, urteilen, wurden allerdings nur sehr wenige Online-Übungen eingestuft, und zwar 3,1\%, was darauf hinweist, dass mit den existierenden Online-Übungen kaum höhere Lernziele erreicht werden können. bedienten sich die Verleger einer ähnlichen Struktur wie bei den Lehrwerken im Papierformat. Die Navigation auf den Webseiten ist aufgrund der Ähnlichkeit mit dem Lehrwerk im Papierformat zumeist sehr benutzerfreundlich und übersichtlich. Für einen Lernenden, der mit dem Lehrwerk nicht vertraut ist, sind lehrwerkbegleitende OnlineÜbungen allerdings weniger von Nutzen, da sie inhaltsabhängig sind und dem Lehrwerkkonzept unterliegen.

Beim Aspekt Übungsformate, die anhand der von den Lernenden durchgeführten Tätigkeit festgelegt wurden, ergab die Analyse der Online-Übungen vier verschiedene Übungstypen. Diese wurden Auswählen, Ergänzen, Verbinden, Einordnen benannt und beschreiben, was Lernende tun sollen, um die Übung zu lösen. Da nur vier Übungstypen vorkommen, kann nicht von einem vielfältigen medialen Lernen gesprochen werden, was bereits von Beatty /10/ beanstandet wurde. Die Potenziale des Web sind diesbezüglich noch sehr groß und was die Übungsformate angeht, wahrscheinlich auch noch nicht erschöpft.

Die Analyse unter dem Aspekt Förderung der Sprachfertigkeiten deutet darauf hin, dass die Coden: IORME7 
große Mehrheit der Online-Übungen Grammatik- und Vokabellernen unterstützt und zum Teil nur auf den behavioristischen Prinzipien basiert. Auch bei den Übungen, die auf den ersten Blick komplexeres Wissen von den Lernenden verlangen, wie z.B. beim Leseverstehen oder Hörverstehen, wird das leider nicht realisiert. Den Fertigkeiten Schreiben und Sprechen wird nur sehr wenig Aufmerksamkeit gewidmet; sie können anhand von Online-Übungen nicht ausreichend gefördert werden. Will man Sprachhandlungen in diesen zwei Bereichen fördern, muss nach anderen Alternativen gesucht werden, denn lehrwerkbegleitende Online-Übungen können hier keine Hilfe leisten.

Die Mehrheit der Online-Übungen basiert auf einem automatischen, vorprogrammierten Feedback. Die Lernenden bekommen sofort eine Antwort darauf, ob sie eine Übung richtig oder falsch gelöst haben. Dass ein Feedback bei den Sprech- und Schreibübungen noch nicht völlig automatisiert werden kann, ist wahrscheinlich ein Grund dafür, dass diese Fertigkeiten fast ausgelassen werden. Gerade beim Üben von Sprechen und Schreiben könnten die Potenziale des semantischen Web 3.0 oder sogar des Web 4.0 in der Zukunft einen wichtigen Beitrag leisten. Das jetzige digitale Angebot kann dies allerdings noch nicht gewährleisten.

Auch hinsichtlich der Lernziele lieferte die Analyse der Online-Übungen keine viel besseren Ergebnisse. Die große Mehrheit der Übungen deckt Lernziele $a b$, die den ersten beiden Stufen nach Bloom - Wissen und Verständnis - zugeordnet werden, die letzten drei Stufen, die komplexeres Wissen erzielen, sind sehr unterrepräsentiert.

Obwohl es scheint, dass klassische Lehrwerke im Buchformat an ihre Grenzen gestoßen sind, können anhand von analysierten OnlineÜbungen keine Schlussfolgerungen darüber gezogen werden, dass das digitale Angebot im Vergleich zu den Lehrwerken im Papierformat einen echten didaktischen Mehrwert hat oder neue Lernmöglichkeiten schafft, die wesentlich anders sind als solche, die bereits im Papierformat bekannt sind.

Die Suche nach entsprechenden digitalen Materialien und deren Didaktisierungen ist sehr zeitaufwändig, manchmal scheint es sogar, dass gerade dieses enorme und unüberschaubare Angebot den Einsatz solcher Materialien in der Praxis zum Scheitern bringt. Legutke /11/ betont, dass das digitale Angebot nur dann zur Geltung kommen und sich als effektiv erweisen kann, wenn bei dessen Einsatz die zentralen Fragen der Fremdsprachendidaktik nicht vergessen werden. Wenn digitale Lernmaterialien nur wegen der allgemeinen Euphorie rund um das Lernen mit den Medien eingesetzt werden, ohne sich dabei Gedanken darüber zu machen, ob sie wirklich den Lernprozess fördern und zum Lernerfolg beitragen können, dann ist diese Herangehensweise als sehr problematisch anzusehen und hat mit den geltenden methodisch-didaktischen Prinzipien des modernen Fremdsprachenunterrichtens nichts $\mathrm{zu}$ tun. Von künftigen DaF-Lehrenden sind infolgedessen hochentwickelte Reflexions- und Medienkompetenzen zu erwarten, die ihnen ermöglichen, die potenzielle Qualität der Online-Übungen bzw. ihren didaktischen Mehrwert $\mathrm{zu}$ bewerten und über den sinnvollen Einsatz von Online-Übungen nachzudenken. Sie werden nämlich aus der großen Menge der existierenden OnlineLernmaterialien passende auswählen oder eigene erstellen müssen. Zusätzlich müssen sie den Lernenden laut Launer /12/ auch die nötigen Strategien beibringen, um das ELearning wirklich effektiv zu gestalten. So wie Rösler /13/ sind auch wir der Überzeugung, dass Lehrwerke in der Zukunft noch stärker modularisiert werden und von den Lehrkräften nach eigenem Bedarf aus einzelnen Teilen zusammengestellt werden. Lehrwerkbegleitende Online-Angebote müssten sehr gut durchdacht werden, damit die Lernenden sie als eine für ihr Lernen effiziente Herausforderung empfinden und nicht hauptsächlich als Marketing-Strategie zur Werbung für ein Lehrwerk. 
Notes

/1/ Kerres, Michael (2001), Multimediale und telemediale Lernumgebungen. Konzeption und Entwicklung. München: Oldenbourg Verlag.

/2/ Mitschian, H. (2010). m-Learning - die neue Welle? Mobiles Lernen für Deutsch als Fremdsprache. Kassel: University Press.

/3/ Beatty, K. (2010). Teaching and researching computer-assisted language learning. Longman, Harlow, UK.

/4/ Mitschian, H. (2010). m-Learning - die neue Welle? Mobiles Lernen für Deutsch als Fremdsprache. Kassel: University Press.

/5/ Szerszeń, P. (2014). Aktuelle Tendenzen im computerunterstützten (Fach-)Fremdsprachenunterricht. Zeitschrift für Interkulturellen Fremdsprachenunterricht 19: 1, 250-260.

/6/ Biebighäuser, K, Zibelius, M., Schmidt, T. (2012). Aufgaben 2.0 Aufgabenorientierung beim Fremdsprachenlernen mit digitalen Medien. In: Biebighäuser, K, Zibelius, M., Schmidt, T (Hrsg.). (2012). Aufgaben 2.0. Konzepte, Materialien und Methoden für das Fremdsprachenlehren und -lernen mit digitalen Medien. Tübingen: Narr (Giessener Beiträge zur Fremdsprachendidaktik).

/7/ Szerszeń, P. (2014). Aktuelle Tendenzen im computerunterstützten (Fach-)Fremdsprachenunterricht. Zeitschrift für Interkulturellen Fremdsprachenunterricht 19: 1, 250-260.

/8/ Retelj, A. (2014). Die Beurteilung von Lehrwerken für Deutsch als Fremdsprache anhand des Gemeinsamen Europäischen Referenzrahmens für Sprachen: eine empirische Untersuchung über die Entwicklung der lexikalischen Kompetenz. In: Lah, Meta (Hrsg.). (2014). Skupni evropski jezikovni okvir - navzkrižni pogledi $=$ Cadre européen commun de référence pour les langues - regards croisés. Ljubljana: Znanstvena založba Filozofske fakultete: 61-75.

19/ Bloom, B.S. (Hrsg.), Engelhart, M.D., Furst, E.J., Hill, W.H., \& Krathwohl, D.R. (1956). Taxonomy of educational objectives: The classification of educational goals. Handbook 1: Cognitive domain. New York: David McKay.

/10/ Beatty, K. (2010). Teaching and researching computer-assisted language learning. Longman, Harlow, UK.

/11/ Legutke, M. (2008). Alte und neue Medien im fremdsprachlichen Klassenzimmer: Discourse-
Szenario - Task. In: Müller-Hartmann, A.

Aufgabenorientiertes Lernen und Lehren mit Medien: Ansätze, Erfahrungen, Perspektiven in der Fremdsprachendidaktik. Frankfurt am Main, Berlin, Bern, Wien [u.a.]: Lang. 65-84.

/12/ Launer, R. (2010). Blende(n)d Deutsch lernen? Ein Blended-Learning-Modell für den Fremdsprachenunterricht. Info DaF 4, 426-435.

/13/ Rösler, D. (2012). So echt wie möglich und/oder so tun als ob? Aufgaben im Kontext sich verändernder Privatheitskonzepte. In: In: Biebighäuser, K, Zibelius, M., Schmidt, T (Hrsg.). (2012). Aufgaben 2.0. Konzepte, Materialien und Methoden für das Fremdsprachenlehren und -lernen mit digitalen Medien. Tübingen: Narr (Giessener Beiträge zur Fremdsprachendidaktik). 91-117.

Literatur

Primarquellen

Online-Programm Kahoot: https://kahoot.it/

(Zugriffsdatum: 11. 2. 2020)

Online-Übungen zum Lehrwerk Optimal:

https://www.klett-sprachen.de/optimal/r-

1/197\#reiter=titel\&niveau=A1 (Zugriffsdatum: 5. 2. 2020).

Online-Übungen zum Lehrwerk Studio express:

https://www.cornelsen.de/codes/products/97830654 99705/gekahe (Zugriffsdatum 4. 2. 2020).

Online-Übungen zum Lehrwerk Ideen:

https://www.hueber.de/shared/uebungen/ideen. (Zugriffsdatum: 26. 1. 2016).

Online-Übungen zu dem Lehrwerk Themen aktuell: https://www.hueber.de/shared/uebungen/themenaktuell/lerner/uebungen. (Zugriffsdatum: 5. 2. 2020). Webseite des Rokus Verlags:

http://www.srednja.net (Zugriffsdatum: 26. 1. 2020). Online- Übungen zum Lehrwerk Klasse!:

https://www.klett-sprachen.de/klasse/r-

1/556\#reiter=titel\&niveau=A1 (Zugriffsdatum: 5. 2. 2020).

Lehrwerkbegleitende Online-Übungen zu Panorama:

https://www.cornelsen.de/codes/products/97830612 04723/guwifu (Zugriffsdatum: 6. 2. 2020).

Das Autorenprogramm Hotpotatoes. : http://hot-

pot.uvic.ca. (Zugriffsdatum: 5. 2 2020). 